Revue internationale P.M.E.

Économie et gestion de la petite et moyenne entreprise

\title{
L'élaboration de savoirs actionnables en PME légitimés dans une conception des sciences de gestion comme des sciences de l'artificiel
}

\section{Marie-José Avenier}

Volume 17, numéro 3-4, 2004

URI : https://id.erudit.org/iderudit/1008462ar

DOI : https://doi.org/10.7202/1008462ar

Aller au sommaire du numéro

Éditeur(s)

Presses de l’Université du Québec

ISSN

0776-5436 (imprimé)

1918-9699 (numérique)

Découvrir la revue

Citer cet article

Avenier, M.-J. (2004). L'élaboration de savoirs actionnables en PME légitimés dans une conception des sciences de gestion comme des sciences de l'artificiel. Revue internationale P.M.E., 17(3-4), 13-42. https://doi.org/10.7202/1008462ar
Résumé de l'article

Deux conceptions de la recherche en PME, également légitimes, coexistent actuellement. Selon la première, la recherche vise à élaborer des savoirs scientifiques sur la gestion des PME prises comme objet d'étude. Selon la seconde, elle vise à élaborer des savoirs pour la gestion des PME, c'est-à-dire des savoirs que des dirigeants de PME pourraient exploiter dans leurs décisions d'action. Ces deux finalités, qui sont souvent opposées, ne peuvent-elles vraiment pas être conciliées ? À quelles conditions des savoirs élaborés pour être exploitables par des dirigeants de PME peuvent-ils être considérés comme légitimés scientifiquement ? Certaines méthodes de recherche sont-elles mieux adaptées que d'autres pour élaborer de tels savoirs ? 


\section{L'élaboration de savoirs actionnables en PME légitimés dans une conception des sciences de gestion comme des sciences de l'artificiel}

Marie-José AVENIER*

CNRS, Université de Lyon III

MOTS CLÉS

\section{PME - Savoir actionnable - Légitimation de savoirs Constructivisme - Sciences de l'artificiel - Méthodes de recherche Recherche-intervention - Étude de cas - Récit de pratiques}

\section{RÉSUMÉ}

Deux conceptions de la recherche en PME, également légitimes, coexistent actuellement. Selon la première, la recherche vise à élaborer des savoirs scientifiques sur la gestion des PME prises comme objet d'étude. Selon la seconde, elle

\section{L'AUTEURE}

Marie-José Avenier est directrice de recherche CNRS à Euristik (CNRS, Université de Lyon III). À la suite d'une double formation disciplinaire (mathématiques et sciences économiques) à la fois en France et aux États-Unis (Normale Sup' Fontenay et Berkeley), elle s'est engagée dans la recherche en sciences de gestion. Ses travaux, qui portent sur le pilotage stratégique des organisations et sur la méthodologie et l'épistémologie de la recherche en sciences de gestion, l'ont conduite à expérimenter au cours des 20 dernières années diverses méthodes de terrain dans des organisations de tailles diverses, de la grande entreprise à la PME. Adresse : Euristik, Université de Lyon III, B.P. 0638, 69239 Lyon, Cédex 02, France. Courriel : <avenier@free.fr.>.

* L'auteure tient à remercier les évaluateurs anonymes qui ont encouragé la publication de cette recherche d'ordre épistémologique, ainsi que le comité de rédaction de la RIPME d'avoir pris le risque d'engager ce débat fondamental pour la recherche en PME, qui, comme les autres domaines de la recherche scientifique, ne peut plus se priver d'une pratique continuelle de «critique épistémologique interne». 
vise à élaborer des savoirs pour la gestion des PME, c'est-à-dire des savoirs que des dirigeants de PME pourraient exploiter dans leurs décisions d'action. Ces deux finalités, qui sont souvent opposées, ne peuvent-elles vraiment pas être conciliées ? À quelles conditions des savoirs élaborés pour être exploitables par des dirigeants de PME peuvent-ils être considérés comme légitimés scientifiquement? Certaines méthodes de recherche sont-elles mieux adaptées que d'autres pour élaborer de tels savoirs?

\section{ABSTRACT}

Two visions of research about SMEs, equally legitimate, presently co-exist. In the first one, research aims at developing scientific knowledge on the management of SMEs taken as research objects. In the second one, research aims at building actionable knowledge for SMEs management, i.e. knowledge that can be used by SME managers in their decision-making process. These two aims are often opposed in academic debates. Is there really no way to conciliate them? Under which conditions knowledge built to be used by SME managers can be considered as scientifically legitimated? Are some research methods better suited than others to build such knowledge?

\section{RESUMEN}

Actualmente existen dos concepciones de investigación acerca de la Pequeña y Mediana Empresa (PyME), ambas legítimas. La primera se concibe como la ciencia que tiene como finalidad el desarrollo de conocimientos científicos sobre la gestión de las PyMEs. La segunda se concibe como la ciencia que tiene por finalidad la elaboración de conocimientos para la gestión de las PyMEs. En otras palabras nos referimos a los conocimientos que los directores de empresas podrían desarrollar y explotar durante el proceso de toma de decisiones. ¿Podrían conciliarse estas dos finalidades que normalmente las encontramos como opuestas? ¿Qué conocimientos utilizados por los directores de empresas podrían considerarse como legítimamente científicos? ¿Qué métodos de investigación serían más adaptados para elaborar tales conocimientos?

\section{ZUSAMMENFASSUNG}

In der Forschung rund um die KMU existieren zur Zeit zwei verschiedene Ansätze. Der erste Ansatz betrachtet die KMU als ein wissenschaftliches Betätigungsfeld, welches die Herausarbeitung von Kenntnissen über das Management von KMUs zur Zielsetzung hat. Die Zielsetzung des zweiten wissenschaftlichen Ansatzes besteht darin, Kenntnisse für das Management von KMU Betrieben zu gewinnen, welche von Führungsverantwortlichen auch direkt in Entscheidungsfindungen eingebracht werden können. Ist es nicht möglich, diese zwei verschiedenen Ansätze, die sich oft gegenüberstehen, in eine übereinstimmende Form zu bringen? Unter welchen Voraussetzungen kann Wissen, das für Führungskräfte ausgearbeitet wurde, als wissenschaftlich legitim betrachtet werden? Gibt es dabei Forschungsmethoden, die sich besser eignen als andere, um Kenntnisse für das Management von KMU zu erlangen? 


\section{Introduction}

Deux conceptions des sciences de gestion également légitimes coexistent actuellement. La première les conçoit comme des sciences ayant pour finalité d'élaborer des savoirs sur la gestion des organisations socioéconomiques prises comme objet d'étude. Certains des savoirs ainsi produits peuvent apparaître comme des savoirs actionnables (Argyris, 1993) pour des professionnels concernés par la gestion et le développement des organisations, principalement des managers ou des décideurs économiques ou politiques. La seconde les conçoit comme des sciences ayant pour finalité d'élaborer des savoirs pour la gestion des organisations sociales, c'est-à-dire des savoirs que des managers ou des consultants pourraient exploiter dans leurs décisions d'intervention au sein d'organisations concrètes.

Ces deux finalités de la recherche, élaborer des savoirs considérés comme scientifiques par la communauté des chercheurs et élaborer des savoirs considérés comme exploitables par des praticiens pour décider de leur action, sont souvent opposées dans les débats sur la vocation des sciences de gestion (Déry, 1997). Ces deux finalités ne peuvent-elles pas néanmoins être conciliées? À quelles conditions des savoirs élaborés pour être exploitables par des praticiens peuvent-ils être considérés comme légitimés scientifiquement? Qu'entend-on exactement par savoirs actionnables en PME? Comment élaborer de tels savoirs ? Certaines méthodes de recherche sont-elles mieux adaptées que d'autres?

La présente contribution a pour projet de rendre compte d'une recherche d'ordre épistémologique menée autour de ces questions, en s'inscrivant dans la seconde conception des sciences de gestion évoquée ci-dessus et dans le prolongement de divers travaux précurseurs (notamment Daft et Lewin, 1990 ; Martinet, 1990 ; d'Amboise, 1996). Trois types de destinataires potentiels de l'élaboration de savoirs actionnables en PME sont privilégiés : les chercheurs en sciences de gestion, les décideurs exerçant leur activité au sein de PME et les consultants intervenant particulièrement dans les PME.

Sur le versant empirique qui est destiné à s'assurer que la réflexion ne s'effectue pas en lévitation par rapport au terrain, cette recherche s'appuie sur l'expérience de nombreuses années de pratique de différentes méthodes de terrain ${ }^{1}$ ainsi que sur les résultats de deux séries d'entretiens exploratoires menées à quatre années d'intervalle auprès de dirigeants d'entreprises et de consultants (Avenier, 2002). Elle prend aussi appui sur les résultats d'un travail interactif entre des praticiens et des chercheurs, réalisé entre les deux enquêtes destinées à approfondir les réponses qui avaient été apportées à la première série d'entretiens. Ces entretiens visaient

1. Dont, notamment, une recherche monographique réalisée en 2003 avec le professeur Claude Pellegrin au sein d'une PME du secteur du transport et de la logistique, de laquelle a été tiré un « cas de stratégie en PME» (Avenier et Pellegrin, 2004). 
notamment à vérifier si les savoirs considérés comme actionnables par ces praticiens de la stratégie avaient des formes particulières, à recueillir leur opinion sur «l'actionnabilité » de la recherche en stratégie et à relever un certain nombre de thèmes de recherche susceptibles d'intéresser des praticiens et sur lesquels on pourrait développer des savoirs actionnables.

L'article s'articule en deux parties. La première s'attache à définir la notion de savoirs considérés comme actionnables par des managers ou des consultants et légitimés dans le référentiel épistémologique des constructivismes. La seconde partie discute des modalités concrètes d'élaboration de savoirs actionnables en PME ou en entrepreneuriat légitimés.

\section{Dans le paradigme des sciences de l'artificiel, qu'est-ce qu'un « savoir actionnable légitimé »?}

Après avoir précisé la distinction adoptée dans cette contribution entre savoir et connaissance, nous mettrons en évidence la pertinence du paradigme des sciences de l'artificiel et du référentiel épistémologique des constructivismes pour appréhender les sciences de gestion. Puis, sera abordée la question de la légitimation des savoirs élaborés, ce qui permettra alors de préciser la signification dans ce référentiel de la notion de savoirs actionnables légitimés. Enfin, la dernière section rendra compte de l'opinion des praticiens que nous avons interrogés concernant la forme et le contenu d'énoncés qu'ils considèrent être potentiellement actionnables.

\subsection{La distinction entre savoirs et connaissances}

Alors que le substantif verbal savoir renvoie généralement au résultat d'un processus, le terme connaissance renvoie aussi bien au processus de connaissance qu'au résultat de ce processus à un instant donné. Convient-il de distinguer ces deux termes ou peut-on les considérer comme synonymes?

Dans les sciences de l'éducation, les notions de savoir et de connaissance (-résultat) sont distinguées (Legroux, 1981). La connaissance est décrite comme un processus se développant dans et par l'expérience. Par définition, la connaissance-résultat fait corps avec l'individu, ce qui la rend ineffable en tant que telle. Le savoir, quant à lui, exprime sous forme de représentations communicables la connaissance qui est intérieure à des sujets. Le savoir se situe donc en quelque sorte à l'interface entre la connaissance, qui est intérieure au sujet, et l'information, qui exprime ce savoir à l'extérieur du sujet. Cette distinction entre savoir et connaissance, que nous adopterons dans le présent article, est au cœur de la problématique de la communication de savoirs et de connaissances en vue de leur appropriation. Elle correspond grosso modo à la distinction entre connaissance tacite et savoir explicite discutée par Nonaka (1994). 
Remarquons que si l'on peut effectivement distinguer ces deux notions, en pratique, savoirs et connaissances sont intimement liés. La position d'interface qu'occupe le savoir entre la connaissance ineffable et sa représentation extérieure au sujet les rend même inséparables, à l'image de l'iceberg, plus ou moins enfoncé et donc plus ou moins visible, le visible étant toujours inséparable de l'invisible. La connaissance ne se développe pas dans le « vide», mais en relation avec des savoirs. Inversement, les savoirs sont toujours rattachés à des connaissances incorporées. Et toute intervention sur l'un affecte l'autre.

\subsection{Les sciences de gestion : des sciences d'ingénierie des organisations socioéconomiques}

Constatant que le monde dans lequel nous vivons est aussi artificiel que naturel, Simon a commencé à conceptualiser dès 1969 ce qu'il a appelé les sciences de l'artificiel ou sciences de conception. Dans cette conceptualisation, il souligne que les objets artificiels qui nous entourent, qu'il dénomme artefacts, ne sont pas hors de la nature et qu'ils n'ont nulle dispense pour ignorer ou violer les lois de la nature ; en même temps, ils présentent la particularité de s'adapter aux buts et aux intentions de l'homme. Il argumente aussi que les questions d'artificialité et de complexité sont inextricablement imbriquées. Ainsi, les sciences de l'artificiel ont été conçues pour permettre de prendre en compte à la fois les finalités en jeu dans les artefacts étudiés et l'éventuelle complexité de ces artefacts et des contextes dans lesquels ils opèrent et évoluent.

Le caractère artificiel des organisations sociales, qu'il s'agisse de PME, de grandes entreprises ou d'administrations, n'est plus à argumenter. Elles sont présentées comme «des construits sociaux finalisés » selon la formule bien connue de Crozier et Friedberg (1977) et comme des «phénomènes dans lesquels s'incarnent à la fois les intentions humaines et les lois naturelles » (Simon, 2004, p. 29) : elles naissent sous l'impulsion de leurs fondateurs ${ }^{2}$ et se développent en référence à certaines intentions et finalités, dans des conditions et dans des environnements que les membres de l'organisation ne maîtrisent pas totalement. Elles mettent en interaction des êtres humains qui ne sont pas des objets passifs et inertes, mais des agents cognitifs et affectifs actifs, dotés de conscience, de capacité de réflexion,

2. «Ce qui donne naissance à une organisation, c'est avant tout la volonté humaine, qu'elle émane d'un individu ou d'un groupe d'individus », réaffirme avec force Hernandez (1999, p. 522) en conclusion de sa relecture de l'évolution de la recherche en entrepreneuriat. Cette étude fait apparaître qu'après s'être presque exclusivement consacrée aux caractéristiques de l'entrepreneur ( $c f$. par exemple Filion, 1991), la recherche en entrepreneuriat, en se développant depuis quelques années autour de la notion d'organisation émergente, risque de tomber dans l'excès inverse qui consiste à attribuer au créateur d'entreprise un rôle mineur. 
d'autofinalisation, d'imagination, d'invention, ayant mémoire, désirs, projets, émotions, etc. Les sciences de gestion peuvent donc être considérées comme des sciences d'ingénierie des organisations socioéconomiques.

Le cadre épistémologique dans lequel ont été élaborées depuis trois siècles la plupart des sciences, en particulier les sciences du naturel et la démarche exclusive qui leur est associée, apparaît mal adapté aux sciences de gestion, quelles que soient les finalités qu'on leur attribue, mais plus particulièrement lorsqu'il s'agit d'élaborer des savoirs pour l'action organisationnelle. En effet, la démarche scientifique classique, qui, schématiquement, procède par étude de l'objet, puis élaboration d'une théorie, puis formulation d'hypothèses à partir de cette théorie et, enfin, test de ces hypothèses, ne convient pas à l'étude de phénomènes influencés par des intentions se déployant en leur sein ou sujets à des émergences inattendues, tels ceux se déroulant dans les organisations sociales. Les phénomènes organisationnels sont façonnés par les interventions finalisées d'acteurs dotés d'imagination et qui, pour atteindre leurs fins, sont susceptibles d'inventer des comportements qui n'auront peut-être pas été prévus dans la conception des tests de la théorie qui visent à la mettre à l'épreuve.

La restauration du paradigme des sciences de l'artificiel (appelées sciences du génie aux $\mathrm{XV}^{\mathrm{e}}$ et $\mathrm{XVI}^{\mathrm{e}}$ siècles, et les arts mécaniques aux $\mathrm{XII}^{\mathrm{e}}$ et $\mathrm{XIII}^{\mathrm{e}}$ siècles) permet de prendre en compte l'influence des finalités potentiellement multiples qui façonnent le comportement et l'évolution des artefacts et la complexité potentielle de ces artefacts et des contextes dans lesquels ils fonctionnent et évoluent. Associé au référentiel épistémologique des constructivismes (Le Moigne, 1995, 2001, 2002), ce paradigme offre aux sciences de gestion un cadre propice dans lequel elles peuvent se déployer ${ }^{3}$.

Examinons maintenant dans quelles conditions ce cadre permet de concilier les deux vocations possibles de la recherche en sciences de gestion: élaborer des savoirs reconnus comme valables à la fois dans la communauté universitaire et dans celle des praticiens.

\subsection{Légitimation plutôt que validation des savoirs}

Dans les épistémologies positivistes, des savoirs sont considérés comme valables lorsqu'ils ont été testés empiriquement selon la démarche rappelée précédemment et que les divers tests d'hypothèses formulées à partir de ces savoirs n'ont pas permis de réfuter ces hypothèses. Ces savoirs sont alors déclarés validés.

3. Le lecteur intéressé par une argumentation détaillée de cette assertion peut consulter diverses références parmi lesquelles : Le Moigne (1990, 1995, 2001); Martinet (1990); Avenier (1997) et David, Hatchuel et Laufer (2000). 
Quand des savoirs sont-ils considérés comme valables dans le référentiel épistémologique des constructivismes?

La théorie de la connaissance dans les épistémologies constructivistes s'inscrit dans la vision kantienne selon laquelle les choses sont inconnaissables telles qu'elles sont en elles-mêmes, en dehors de l'expérience sensible ou cognitive. Plus précisément (Le Moigne, 1995, p. 72), elle pose que «le réel connaissable est un réel en activité qu'expérimente le sujet, et que ce sujet se construit, par des représentations symboliques (schémas, lettres, chiffres, phonèmes, etc.), cette connaissance de son expérience du réel». Elle pose également le caractère téléologique de la connaissance, «le phénomène modélisé est connu finalisé par l'action cognitive de sa représentation» (ibid., p. 76) et «l'irréversibilité de la flèche du temps» (ibid., p. 73). La connaissance est alors vue comme un processus de construction intentionnelle de représentations éprouvées par l'expérience.

Alors que, pour cause d'irréversibilité temporelle, les observations effectuées dans une organisation et plus encore les formes d'expérimentations réalisées dans le cadre de recherches-actions ne sont pas reproductibles, les processus cognitifs mis en œuvre en vue de l'élaboration des savoirs, quant à eux, sont reproductibles. C'est sur cette propriété importante que s'appuie la validation des connaissances dans les épistémologies constructivistes. Mais plutôt que de validation, nous préférons parler, avec Le Moigne (1995), de légitimation des savoirs. Dans un contexte scientifique, le terme de validation prend en effet une signification bien particulière, celle de tester empiriquement dans le cadre d'une démarche hypothéticodéductive, alors que, comme nous allons le voir maintenant, dans le référentiel épistémologique des constructivismes, la légitimation des savoirs suit une autre voie.

Des savoirs seront considérés comme légitimés lorsqu'ils auront résisté non plus aux assauts de multiples tests empiriques comme dans les positivismes, mais, comme le suggérait Piaget dès 1967, aux assauts d'une critique épistémologique rigoureuse sur les processus d'élaboration de ces savoirs, réalisée tout au long de la recherche par le chercheur lui-même ainsi que, possiblement, ex post par toute personne s'intéressant aux savoirs ainsi élaborés, quel que soit son statut professionnel : chercheur ou praticien.

La volonté de légitimer dans leur contexte les savoirs qu'il élabore impose donc au chercheur d'expliciter continuellement pour lui-même les processus cognitifs qu'il met en œuvre dans sa recherche et de s'assurer de leur cohérence et de leur pertinence eu égard au projet de connaissance poursuivi et aux expériences sensibles et cognitives qui nourrissent ces processus cognitifs. Cette démarche rendra ensuite possible la critique épistémologique externe.

Pour permettre la critique épistémologique externe, «le chercheur est tenu de rendre explicite l'ensemble des éléments permettant de discuter des énoncés 
produits » (Giordano, 2003, p. 17) : explicitation des principaux présupposés ; description des cheminements; présentation des informations mobilisées et des conditions dans lesquelles ces informations ont été obtenues ${ }^{4}$ (Plane, 1998) ; argumentation des allers et retours effectués entre questions de recherche, référents théoriques mobilisés et informations recueillies sur le terrain; explicitation des référents théoriques finalement retenus et justification de leur pertinence et de leur cohérence mutuelle ainsi qu'avec les informations recueillies, avec les modes de traitement de ces informations et avec le positionnement épistémologique adopté, etc. Autrement dit, «l'exigence de "traçabilité" y est très forte car plus difficile à remplir que dans les démarches plus cadrées » (Giordano, 2003, p. 12).

Comme le précise Martinet (2000), la critique épistémologique interne (c'est-àdire effectuée par le chercheur lui-même), également appelée travail épistémique, ne porte pas seulement sur le processus cognitif d'élaboration de ces savoirs : elle s'adresse également aux produits de ce processus, c'est-à-dire aux savoirs élaborés eux-mêmes. Elle consiste à questionner le sens profond des notions mobilisées et / ou élaborées, à les mettre en perspective théorique et pratique, à les articuler aux savoirs déjà admis et éventuellement à procéder à leur déconstruction-reconstruction.

Ainsi, avec Martinet (2002), nous pouvons considérer que «le travail épistémique est aux constructivismes ce que la démarche scientifique est aux positivismes », en soulignant néanmoins une différence notable: contrairement aux épistémologies positivistes où l'élaboration des savoirs et la validation de ces savoirs s'effectuent au cours de deux processus consécutifs dissociés, dans les épistémologies constructivistes, les processus d'élaboration et de légitimation des savoirs sont simultanés et indissociables. Le statut des savoirs ainsi élaborés est celui d'hypothèses plausibles. Faisant office de «bâtons d'aveugle» (Hafsi, 1997, p. 19), ils fournissent des repères, des « pistes de raisonnement, des avenues possibles à exploiter» (d'Amboise, 1996, p. 29), des «opérateurs de sens » (Martinet, 1997, p. 74), qui peuvent être mobilisés pour «bien conduire sa raison dans les affaires humaines » (Simon, 1983) et « agir en plus grande sûreté intellectuelle» (Martinet, 1990, p. 231).

Nous illustrerons ces propos abstraits avec deux exemples particuliers, choisis parmi tant d'autres. Premier exemple : les repères proposés pour l'identification des dysfonctionnements d'une organisation dans la méthode de diagnostic socioéconomique développée par l'ISEOR sur la base de près d'un millier de cas de recherches-interventions menées en 25 ans dans des organisations dont les caractéristiques de taille, de secteur d'activité, de statut juridique, etc., sont extrêmement variées (Savall et Zardet, 1996, p. 175-176). Cette méthode explicite

4. Est-ce au cours d'entretiens ? Expriment-elles des changements constatés à la suite d'interventions conçues et mises en œuvre au cours d'une recherche-action ? ... 
six points sensibles ${ }^{5}$ à examiner systématiquement dans un diagnostic, outre la recherche de dysfonctionnements spécifiques à chaque organisation. Second exemple : les concepts d'entreprise-réseau (ou entreprise partagée) et de synergie créatrice et les conditions facilitantes pour la mise sur pied d'une entreprise-réseau ${ }^{6}$, qui ont été élaborés par la Chaire Bombardier en s'appuyant sur 10 années de recherches-interventions au sein d'un même groupement d'entreprises, à savoir l'entreprise-réseau que constitue Bombardier Produits récréatifs et une vingtaine de ses PME sous-traitantes ${ }^{7}$ (Julien et al., 2003).

\subsection{Dans un référentiel constructiviste, qu'est-ce qu'un savoir actionnable légitimé?}

La notion de savoir actionnable ${ }^{8}$ a été définie par Argyris en 1993 comme «un savoir à la fois valable et pouvant être " mis en action" dans la vie quotidienne » (p. 257). Un «savoir valable» est, pour cet auteur, un savoir qui a été validé (au sens positiviste), «le test principal consistant à formuler des "énoncés de vérité » sous forme d'hypothèses, puis à essayer de les réfuter au lieu de chercher seulement à les confirmer », sachant qu'il s'agit de "vérité avec un petit “v"» (p. 294) et «d'une quête de la vérité au service du perfectionnement» (p. 293).

5. Ces six points sont: les conditions de travail, l'organisation du travail, la gestion de temps, la communication-coordination-concertation, la formation intégrée et la mise en œuvre stratégique.

6. Ces conditions facilitantes sont (cf. op. cit., p. 101-105) : un changement de philosophie (recentrage sur les métiers de base); un pari sur l'avenir fondé sur une confiance réciproque assise sur la signature d'ententes à moyen terme ; l'existence de proximité géographique sinon psychosociologique; des partenaires ayant un même niveau de développement technologique ; la mise en place d'un processus d'apprentissage partagé ou collectif; le développement d'échanges d'informations riches ; le développement d'une innovation partagée ou diffuse ; des comportements de concurrence-coopération.

7. Sur ce point, voir aussi la contribution dans ce numéro de P.-A. Julien.

8. Le terme actionnable est malheureux, car il a une connotation mécaniste qui va à l'encontre de ce qu'il vise à communiquer: la mise en œuvre d'un savoir actionnable ne se réduit pas à l'application mécaniste de méthodes ou de recettes prêtes à l'emploi. Pour contourner cette difficulté, divers chercheurs ont utilisé d'autres termes. Par exemple, Martinet (2000, p. 111) emploie le qualificatif praticable, qui renvoie au potentiel de mise en pratique de ces savoirs ; Blanco et Lesca (2002), le terme activable, qui donne l'image que l'on va activer un processus (potentiellement complexe) plutôt qu'actionner un levier ou une manette. Ces termes présentant néanmoins divers inconvénients, nous conserverons ici le terme d'origine. 
Argyris avoue par ailleurs (p. 279) ne pas s'accorder totalement avec le point de vue positiviste, mais accepter certains de ses préceptes fondamentaux, notamment les quatre tests du raisonnement causal concernant la vérification de la validité des hypothèses et des explications indiqués par Lee (1989a, 1989b, 1991) : 1) la falsifiabilité du raisonnement, 2) la cohérence logique, 3) l'explicativité et 4) la résistance du raisonnement à la réfutation et à la comparaison avec un raisonnement rival.

On peut s'interroger sur la cohérence épistémique de cette position qui consiste à retenir des critères de validation dont certains s'accordent mal avec le point de vue que l'auteur exprime par ailleurs (respectivement p. 294, 275 et 274) : "l'univers que nous étudions est créé par l'homme», "l'homme est un être de dessein », et le caractère fondamental de la «causalité intentionnelle» (c'est-àdire, l'importance de prendre en compte les finalités humaines dans la compréhension des phénomènes). À cet égard, la formule «il s'agit de vérité avec un petit “v" » est symptomatique du malaise qu'engendre une telle position.

Quoi qu'il en soit, la définition ci-dessus peut être conservée mot à mot pour définir dans le référentiel des constructivismes l'expression «savoir actionnable légitimé » dans tel et tel contexte, à condition évidemment d'attribuer au qualificatif « valable » le sens de « légitimé » tel qu'il a été défini dans le paragraphe précédent.

Il reste à examiner la signification et les implications de la seconde partie de cette définition : un savoir pouvant être « mis en action» dans la vie quotidienne.

Pour qu'un savoir puisse être mis en action dans la vie quotidienne, il est impératif qu'il soit en rapport avec des problématiques que rencontrent dans la vie quotidienne les personnes auxquelles il s'adresse. Mais cela ne suffit pas : encore faut-il qu'il soit non seulement recevable mais aussi effectivement reçu par ces personnes. «Ils [des savoirs] ne sont recevables que si nous pouvons les comprendre, les relier à nos expériences du monde de la vie (en italique dans le texte car traduction du Lebenswelt de Husserl) », nous dit Le Moigne (2000, p. 8-9). Ils seront reçus lorsque les personnes auxquelles ils sont destinés se les seront effectivement appropriés et seront en mesure de les mobiliser à bon escient. Ainsi, comptent non seulement leur contenu, mais aussi la manière dont ces personnes auront été mises en relation avec ces savoirs, qui leur aura ou non permis de se les approprier.

Un savoir en sciences de gestion pouvant être «mis en action» dans la vie quotidienne est un savoir qui peut être mobilisé par des praticiens pour comprendre des situations et/ou intervenir pour améliorer des pratiques, des fonctionnements et, plus généralement, les performances de leur organisation. Il doit donc être d'une certaine manière en rapport avec ces problématiques. En ancrant sa recherche dans 
des terrains d'étude concrets, et en définissant ses questions de recherche à partir de préoccupations concrètes de praticiens, un chercheur accroît ses chances d'élaborer des savoirs qui pourront être considérés par des praticiens comme actionnables.

\subsection{Forme et contenu des savoirs actionnables : le point de vue de praticiens}

Au cours de la première enquête, nous avons interrogé nos interlocuteurs sur la forme et le contenu d'énoncés qui constitueraient pour eux des savoirs actionnables en stratégie. Cela dépend! a été la réponse unanime. Cela dépend de la personne, de sa culture, de ses projets, de ses contextes d'action, de ses préoccupations du moment, etc.

Toutefois, en approfondissant la question, la plupart des praticiens interrogés se sont dit peu réceptifs aux énoncés normatifs ou prescriptifs. Certains se sont déclarés intéressés par les formalisations qui les aident à répondre à des questions qu'ils se posent, non pas en leur indiquant ce qu'il faut faire nécessairement, mais en leur apportant des éclairages sur diverses manières d'agir ou de poser le problème ${ }^{9}$. D'autres apprécient les conceptualisations qui les interpellent ou qui suscitent leur réflexion (par exemple, la notion d'organisation apprenante), ou qui les aident à mieux comprendre ou décoder l'univers dans lequel ils interviennent (par exemple, les trois modèles théoriques de décision mobilisés par Allison, 1971). D'autres sont intéressés par les outils, tels que les matrices de portefeuille d'activités, parce qu'ils les incitent à se poser des questions auxquelles ils n'auraient pas pensé spontanément. Certains, enfin, ont déclaré qu'il leur paraissait plus utile de lire des ouvrages favorisant l'ouverture d'esprit (lectures philosophiques, sur le temps, sur le bouddhisme, etc.) que des livres de management.

Lors de la réunion d'approfondissement, les cadres conceptuels, les schémas de synthèse, les modèles d'intégration ou de distinction globaux, tels que la typologie stratégie délibérée / stratégie émergente (Mintzberg et Waters, 1985) ou les matrices de portefeuille, sont apparus comme des formes de savoir prisées par les consultants présents. Ils les utilisent comme des grilles de lecture pour appréhender des situations et comme des supports de réflexion, de questionnement et de communication pour mettre en perspective les représentations des différents acteurs et leur faire prendre conscience des limites à la fois de leur approche individuelle du problème et de la réflexion menée.

9. Ce constat rejoint la position de d'Amboise (1996, p. 29) : «Leur rôle [le rôle des chercheurs] n'est pas celui de fournir des solutions toutes faites aux entrepreneurs et dirigeants. Ils doivent plutôt apprendre à offrir, avec beaucoup de nuances, des pistes de raisonnement, des avenues possibles à exploiter. » 
In fine, trois formes génériques de savoirs susceptibles d'intéresser les praticiens que nous avons interrogés se dégagent : 1) des apports théoriques fondamentaux, notamment philosophiques, donnant des repères qui aident à structurer la pensée, «à y voir un peu plus clair »; 2) à l'autre extrême, des apports pratiques élaborés à partir d'expériences concrètes, parce qu'ils sont susceptibles d'éclairer des situations qu'ils vivent; 3) des cadres conceptuels ou méthodologiques (tels que, par exemple, le modèle de dynamique concurrentielle de Porter ou les méthodes de portefeuille d'activité). Et lorsqu'on part de questionnements qui les intéressent, les praticiens ne refusent pas de monter en abstraction.

Ils déclarent n'accorder d'intérêt aux travaux intermédiaires, c'est-à-dire ceux dans lesquels le propos développé ne porte ni sur des théories fondamentales ni sur des problématiques concrètes, que si ces travaux assurent l'interface entre une théorie fondamentale et un cas concret. Les théories intermédiaires ne leur paraissent en effet pas suffisamment éclairantes du point de vue conceptuel et pas assez opératoires du point de vue pratique pour leur être utiles dans leur activité professionnelle.

La notion de savoirs actionnables légitimés étant clarifiée, nous pouvons examiner les conditions d'élaboration de tels savoirs dans la recherche en PME et en entrepreneuriat.

\section{Comment élaborer des savoirs actionnables légitimés?}

Avant de porter le projecteur sur la question des modalités pratiques d'élaboration de savoirs actionnables légitimés, nous évoquerons diverses considérations d'ordre théorique. Cette deuxième partie s'achèvera sur une discussion des atouts et des difficultés propres aux différentes méthodes de recherche pour l'élaboration de savoirs actionnables légitimés en entrepreneuriat et pour la gestion des PME.

\subsection{Réponse théorique : la boucle d'apprentissage « faire pour connaître et connaître pour faire "}

Si l'on se réfère à l'hypothèse suivante émise par le philosophe Vico (1708/1981) : «Comme la vérité de Dieu est ce que Dieu connaît en le créant et en l'assemblant, la vérité humaine est ce que l'homme connaît en le construisant lui-même, en le formant par ses actions. Le vrai est le faire même » : on ne connaît vraiment que ce que l'on a fait. Autrement dit, les connaissances pour l'action ne peuvent être développées que dans l'action et par l'action. C'est ce qui conduit Le Moigne (2000, p. 10) à définir la connaissance actionnable comme «la connaissance des faire, dans, par et pour l'action » et d'Amboise (1996, p. 27) à remarquer : «C'est 
dans la dynamique de l'action que les nouvelles “théories " se développent. » Cette position rejoint celle du philosophe pragmaticien américain Dewey (1938/1993), le père du learning by doing.

C'est à partir de considérations proches que, plus près de nous, historiquement et en termes de champ d'expérience, Lewin (1946) a introduit une méthode de recherche nouvelle, la recherche-action. L'hypothèse fondatrice de cette méthode est que l'on ne peut accéder à la connaissance d'un système social qu'en en faisant varier les dimensions constitutives et les relations, c'est-à-dire en induisant un changement de celui-ci. Dans le même esprit, Moisdon (1984, p. 70) soutient l'idée selon laquelle «On ne peut guère analyser le fonctionnement de l'organisation que quand celle-ci se met en mouvement, de même qu'un champ de force dévoile sa structure quand on peut observer les déplacements des objets qui y sont plongés ».

Ces différentes contributions traduisent un changement fondamental de perspective en sciences de gestion dans la relation entre connaissance et pratique. Alors que celles-ci avaient coutume d'inscrire leur projet de connaissance dans la vision connaître pour faire, il s'agit aussi désormais, pour certains chercheurs, de faire pour connaître. Autrement dit, d'inscrire le projet de connaissance dans la dialectique «faire pour connaître et connaître pour faire».

La pratique des retours d'expérience, qui se développe de plus en plus dans les entreprises, s'inscrit aussi dans l'optique d'apprendre à partir de l'action. Les savoirs actionnables ainsi développés viennent s'articuler aux connaissances déjà mobilisables dans l'entreprise. Cette élaboration de savoirs au sein des organisations s'effectue généralement dans un but de capitalisation de connaissances existant dans l'organisation à des fins de partage et d'exploitation ultérieure. Elle ne vise généralement pas l'élaboration de savoirs actionnables légitimés. De ce fait, en général, elle ne s'accompagne pas du travail épistémique indispensable, et même consubstantiel à la légitimation des savoirs actionnables élaborés. Certains qualifient ces savoirs d'ordinaires (notamment, Maffesoli, 1985 ; Calori, 2001), d'autres de pré-scientifiques (Paturel et Savall, 2001). Nous préférons les qualifier de locaux pour souligner le caractère local (situé dans le temps et dans l'espace) de leur légitimité : ils ont pour seule légitimité le fait d'avoir été construits à tel moment, dans telles et telles circonstances, à partir de l'expérience de tels et tels praticiens de telle organisation. Un travail épistémique rigoureux sur ces savoirs locaux et sur les processus par lesquels ils ont été élaborés peut évidemment permettre de construire à partir de ceux-ci des savoirs actionnables légitimés.

Le développement de savoirs actionnables locaux ne se produit pas de manière spontanée ni automatique dans les entreprises. Il prend place lorsque, dans une entreprise, certains praticiens ont la volonté d'expliciter les connaissances tacites qu'ils développent dans le cadre de leur pratique. Dans les PME particulièrement, ce travail d'explicitation est encore rarement effectué faute de moyens et 
de temps, ce qui a amené différents chercheurs (notamment Paturel et Savall, 2001 ; Calori, 2001) à souligner la présence de gisements énormes de connaissances tacites insues, c'est-à-dire dont les détenteurs, les « savants ordinaires » (Girin, 1990, p. 155), ne savent pas qu'ils les ont. Ces connaissances, qui n'ont jamais eu l'occasion d'être explicitées et transformées en savoirs actionnables locaux, ne demandent qu'à être transformées en savoirs actionnables légitimés.

Les réponses de certains des dirigeants de PME interrogés en 1998, qui considèrent comme actionnables certaines contributions philosophiques (pensées de Sun Tzu par exemple) et certaines théories générales parce qu'elles leur fournissent des repères fondamentaux $(c f . \S 1.5)$, donnent à penser qu'il pourrait exister deux voies différentes pour développer des savoirs actionnables. Une première consisterait pour le chercheur à travailler sur des théories générales à partir de catégories idéalisées de représentations de phénomènes; elle est peu sûre au sens où rien n'assure au chercheur qu'il existera des praticiens pour considérer comme actionnables les savoirs qu'il élaborera ainsi. La seconde, plus sûre, serait de mener des recherches dans le cadre de projets conçus à partir de préoccupations concrètes de praticiens ou même à partir de savoirs actionnables locaux. Explorons cette question plus avant.

\subsection{Réponse pratique : pas de méthode de recherche privilégiée}

Comme, par définition, les connaissances pour l'action se développent dans l'action et par l'action, la recherche-action ne pourrait-elle pas constituer une méthode de recherche privilégiée pour développer des savoirs actionnables légitimés ? En vue d'instruire cette question, commençons par situer la recherche-action par rapport aux autres méthodes de recherche disponibles.

\subsubsection{Préliminaire: une typologie des différentes méthodes de recherche}

Selon les auteurs et les contextes, l'expression méthode de recherche ou méthodologie de recherche peut être utilisée pour désigner des procédés se situant à des niveaux très différents en termes de manières d'envisager et d'organiser la recherche, de recueillir des informations, de les traiter, etc. Ainsi, par exemple, l'expression méthode de recherche est parfois utilisée pour désigner la démarche générale : construction de théorie - élaboration d'hypothèses - test des hypothèses. À l'autre extrême, elle peut être employée pour désigner une technique particulière de traitement de données (par exemple, la méthode des correspondances). Compte tenu du projet de cet article, nous nous situerons à un niveau intermédiaire entre 
ces deux extrêmes, dans l'esprit de diverses typologies qui ont déjà été proposées ${ }^{10}$. En combinant ces typologies, cinq méthodes-types (au sens des idéaux-types) peuvent être dégagées. Elles se différencient selon que le processus de connaissance mis en œuvre repose ou non sur l'exploitation systématique d'informations relatives à des organisations concrètes et, dans le premier cas, selon la manière dont sont produites les informations mobilisées. Un projet de recherche peut s'appuyer sur la mise en œuvre d'une seule de ces méthodes-types ou au contraire sur l'utilisation combinée de plusieurs d'entre elles.

1. Les recherches logico-formelles. «Il s'agit de réflexions et de synthèses à partir de documents de recherche, constructions théoriques, inventaires critiques, confrontations de modèles ou de méthodes » (Hlady Rispal, 2002 , p. 33). Le processus de connaissance ne prend pas appui sur l'exploitation systématique d'informations relatives à des organisations concrètes ; il procède essentiellement par conceptualisation et mise en relation de catégories idéalisées de représentations de phénomènes (Avenier, 1985).

2. Les recherches empirico-formelles. Elles se construisent autour de la méthodologie du test d'hypothèses. Les informations sont collectées de façon très structurée sur un terrain ou des terrains spécifiques, ou à partir de bases statistiques existantes (Hlady Rispal, 2002, p. 33), ou encore, dans le cas des recherches expérimentales, dans le cadre d'expériences contrôlées en laboratoire, qui ne visent pas systématiquement à reproduire une situation concrète mais donnent la possibilité d'agir sur quelques variables critiques et dans des conditions bien définies (Avenier, 1989).

3. La recherche-action ou recherche-intervention. La recherche-action « organise une consultation active et méthodique dans l'organisation, pour produire une connaissance sur un processus de changement avivé et activé » (Wacheux, 1996). Elle repose sur le principe fondamental énoncé par Lewin, qui peut être résumé par la formule «transformer la réalité pour la connaître » $(\$ 2.1)$. La recherche-action peut prendre des formes très variées. Ainsi Allard-Poesi et Perret (2003) distinguent non seulement la recherche-action lewinienne, l'action science (Argyris, Putnam et McLain Smith, 1985), la recherche ingénierique (Chanal, Lesca et Martinet, 1997), la recherche intervention (Moisdon, 1984 ; Savall et Zardet, 1996; Plane, 2000; David, 2000), qui s'inscrivent dans la logique changer pour connaître, mais aussi les approches psychosociologiques à

10. Par exemple, Yin, 1984 ; Avenier, 1985, 1989 ; Savall et Zardet, 1996, 2004 ; David, 2000 ; Hlady Rispal, 2002 ; Giordano, 2003.

Revue internationale P.M.E., vol. 17, nos 3-4, 2004 
visée thérapeutique, les approches «militantes», les approches «coopératives », qui visent essentiellement la participation et l'émancipation des acteurs des systèmes sociaux sur lesquels elles sont mises en œuvre. L'étude clinique (Girin, 1981) et la co-génération (Wacheux, 1996) peuvent également être considérées comme s'inscrivant dans la méthodologie générale de la recherche-action.

4. La recherche monographique ou étude de $\operatorname{cas}^{11}$ ou encore rechercheobservation. Tout comme les recherches-actions, les recherches de ce type peuvent prendre de multiples formes. Elles ont en commun deux propriétés : 1) le recueil des informations s'effectue dans une ou plusieurs organisations concrètes; 2) ces méthodes s'appuient fréquemment sur l'étude de transformations et de changements organisationnels, mais, à la différence de la recherche-action, le chercheur n'a pas participé à la définition de ces changements, et, bien souvent, ceux-ci sont antérieurs au démarrage de la recherche. Plus précisément, «L'étude de cas suppose que l'on documente un phénomène en utilisant plusieurs techniques de collecte de données (étude de documents, entretiens, observations, etc.). Ces données d'origines diverses servent à construire le cas qui sera analysé en fonction d'une problématique particulière (Hamel, Dufour et Fortin, 1993). L'étude de cas relate donc un événement ou une série d'événements (présents ou passés) pour en tirer un savoir théorique susceptible d'éclairer la pratique » (Giroux, 2003, p. 45).

5. Les recherches biographiques. Tout comme les méthodes précédentes, elles peuvent aussi prendre des formes très diverses, qui s'articulent néanmoins autour de trois genres différents (Rouleau, 2003) : les autoportraits, les récits de vie et les récits de pratiques. Dans l'autoportrait, le chercheur fait raconter à une personne l'ensemble de sa vie, considérée comme une source d'information importante sur une problématique spécifique. Les récits de vie visent à recueillir les narrations de personnes retraçant un parcours, en s'attachant à la manière dont elles mettent en scène ces événements, les analysent, portent des jugements sur ce qu'elles disent. Le contenu de ces récits peut ensuite être étudié par différentes techniques (biogrammes, analyse thématique, matrices d'événements, élaboration de profils, constitution de mots clés, analyse structurale, etc.), de manière à dégager des noyaux de sens. Les récits de vie peuvent aussi être utilisés pour étudier des phénomènes collectifs tels que «l'esprit d'entreprise

11. L'étude de cas désigne ici une méthode de recherche à part entière, distincte de la «méthode des cas » pratiquée en situation de formation pour illustrer une problématique de gestion sur une description courte et simplifiée d'un exemple de cette problématique dans une entreprise. 
collectif» (Marchesnay, 2002). Dans ce cas, on procède par confrontation des récits de vie d'acteurs diversifiés intervenant dans le collectif considéré. Les récits de pratiques, enfin, visent à faire s'exprimer les narrateurs sur un aspect de leur vécu correspondant à un type de pratiques spécifiques et à recueillir leur vision ou leur «théorie » des événements survenus ou des pratiques étudiées; ils constituent une voie privilégiée pour faire expliciter des connaissances tacites $(c f . \S 2.3 .3)$.

Les trois dernières méthodes-types évoquées sont généralement qualifiées de qualitatives, bien que les matériaux recueillis puissent être aussi bien de nature qualitative (propos recueillis dans des narrations, par exemple) que quantitative (mesures diverses, fréquences, etc.) et puissent faire l'objet de traitements quantitatifs. Elles partagent la propriété spécifique de s'intéresser aux situations «naturelles » et spécifiques plutôt qu'aux régularités a-contextuelles et d'étudier les phénomènes dans leur cadre naturel ordinaire plutôt que dans des conditions artificielles comme dans l'expérimentation en laboratoire.

\subsubsection{Concevoir une méthode adaptée au projet de connaissance et inversement}

À la fin des années 1990, diverses questions concernant l'élaboration de savoirs (en général et pas seulement actionnables) en sciences de gestion, qui ont été sources de confusion et de disputes pendant de nombreuses années, semblent désormais assez bien clarifiées.

- «La complémentarité des méthodes » et le «Le pluralisme méthodologique contrôlé » défendus dès 1985 par quelques chercheurs (Avenier, 1985 ; Martinet, 1990 ; Girin, 1990) semblent être des positions de plus en plus répandues dans la recherche francophone en management (Thiétart et collab., 1999), si ce n'est encore dans l'ensemble des sciences de gestion. Que disait alors Martinet? «Admettons plutôt le pluralisme méthodologique contrôlé - aucune méthode n'a, a priori, le monopole de la rigueur et de la raison - qui veille au travail conceptuel, à l'explicitation de ses présupposés, à la pertinence, à la cohérence et à l'efficacité des modélisations, à la lisibilité des cheminements entre termes théoriques et termes empiriques, à la communicabilité des énoncés » (p. 23).

- Le positionnement des recherches qualitatives par rapport à celui des recherches empirico-formelles a été éclairci ( $c f$. notamment Mbengue, 2001 ; Hlady Rispal, 2002 ; Giordano, 2003). Dans ces dernières, qui privilégient largement la méthode hypothéticodéductive, les cheminements sont relativement cadrés, quasi programmables, puisqu'ils suivent le schéma linéaire : hypothèses-prédictions-observations-tests. Il est désormais 
accepté qu'en revanche une recherche qualitative puisse suivre un cheminement complexe, fait d'allers et retours, de mises en forme flexibles, avec éventuellement réorientation du travail en cours de route, en fonction des contraintes spécifiques du terrain et du développement de la compréhension de la problématique, selon le principe de «l'opportunisme méthodique » défendu dès 1990 par Girin. En contrepartie, il est attendu du chercheur qu'il explicite sa position épistémologique et qu'il argumente ses décisions successives et ses choix méthodologiques, qui doivent être à la fois cohérents avec sa position épistémologique et adaptés au projet de connaissance qu'il poursuit (Girod-Séville et Perret, 1999).

Dans le cas spécifique de l'élaboration de savoirs actionnables, Martinet (2000) défend la thèse d'un contrôle constant du processus de recherche par un double ancrage : l'épreuve du terrain et le travail épistémique. L'importance qu'il donne au terrain ne relève pas d'un a priori épistémologique, mais provient de la spécificité même de la connaissance en sciences de gestion: selon cet auteur, celle-ci «ne peut que placer au centre de ses objets de recherche les problèmes et les situations de gestion tels qu'ils se manifestent concrètement dans leurs contingences et leurs évolutions » (p. 121). Dans cette vision, que Paturel et Savall (2001, p. 194) qualifient de «conception non méprisante du terrain», les entreprises n'apparaissent plus comme des lieux passifs de collecte d'informations destinées à nourrir les questionnements des chercheurs. Les organisations en général, les PME en particulier, sont reconnues comme des lieux où se développent des questionnements fondamentaux sur la gestion, des connaissances tacites et, de plus en plus souvent, des savoirs actionnables locaux. À cet égard, d'Amboise (1996, p. 27) considère que «Les dirigeants de PME peuvent en apprendre au chercheur » et Jelinek et Litterer (1995, p. 164) que «La science des organisations est à la traîne des pratiques entrepreneuriales $»^{12}$. Il peut même s'y développer des savoirs actionnables légitimés co-générés par des chercheurs et des praticiens (Wacheux, 1996). Dans ce dernier cas, le projet de recherche, c'est-à-dire la problématique de recherche et la méthode de recherche mise en œuvre, est défini conjointement par des chercheurs et des praticiens.

Le travail épistémique consiste, entre autres, à s'assurer que la problématique et la méthode de recherche choisies sont mutuellement pertinentes et cohérentes entre elles ainsi qu'avec la position épistémologique du chercheur. "C'est de la qualité de l'aller-retour dialectique, dans la cohérence et la pertinence, entre objectif, méthode et analyse, qu'une bonne recherche peut émerger », notent Thiétart et al. (1999, p. 5). Parfois, pour des raisons telles celles évoquées dans la section 2.3, la méthode de recherche prime. La problématique de recherche est alors à adapter

12. «Organizational science lags behind entrepreneurial practice», écrivent-ils. 
pour que la méthode de recherche négociée sur le terrain rende effectivement possible d'accéder aux informations indispensables pour mener à bien le projet de connaissance $^{13}$. Ainsi, par exemple, comme l'indiquent Julien et Marchesnay (1992, p. 29), une recherche sur les processus de prise de décision stratégique dans les PME en fonction du profil du dirigeant, «s'accommode mal d'un questionnement fermé, a fortiori postal. On devrait plutôt s'orienter vers une procédure de rechercheaction, avec l'objectif de contribuer à l'aménagement du PDDS ${ }^{14}$. On touche alors à un aspect important de l'épistémologie des sciences de gestion. »

Par ailleurs, il est essentiel de garder présent à l'esprit que quelle que soit la méthode de recherche adoptée et quelles que soient les techniques de traitement utilisées, donc même s'il s'agit de techniques quantitatives, le statut des savoirs actionnables légitimés par le travail épistémique est celui d'hypothèses plausibles, de repères destinés à enrichir les représentations et les compréhensions possibles d'un phénomène. Les «conditions facilitantes» (Thiétart, 2001, p. 375) éventuellement mises en évidence ne sont jamais à considérer comme des conditions suffisantes ni même nécessaires.

Appliquant ce principe général à la problématique de l'élaboration de savoirs actionnables, il ressort que l'ancrage de la recherche dans un ou des terrains concrets, même s'il facilite le développement de savoirs actionnables, ne peut être considéré comme nécessaire ni comme suffisant pour l'élaboration de tels savoirs. En effet, d'une part, comme nous l'avons mis en évidence dans le $\$ 2.1$, certains savoirs théoriques, qui pourraient par exemple être élaborés dans le cadre de recherches logico-formelles, peuvent apparaître à certains praticiens comme des savoirs actionnables, mais cette voie de recherche est peu «sûre ». D'autre part, pour que des savoirs élaborés à partir d'un travail de terrain puissent être considérés comme actionnables, encore faut-il qu'ils entrent en résonance avec les préoccupations effectives de certains praticiens. Les chances pour qu'il en soit ainsi seront accrues si les questions de recherche étudiées sont construites à partir de problématiques effectivement rencontrées sur des terrains concrets et, plus encore, si ces questions sont définies en interaction avec des praticiens.

En conclusion, toutes les méthodes de recherche, lorsqu'elles sont mises en œuvre dans la perspective spécifique d'élaborer des savoirs actionnables sur des questions qui présentent un intérêt évident pour des praticiens et qu'elles s'accompagnent du travail épistémique indispensable à la légitimation de ces savoirs, peuvent a priori permettre de construire des savoirs actionnables légitimés.

13. Dans le même ordre d'idées, Savall et Zardet (1996) insistent sur l'importance de bien négocier les positions possibles du chercheur, préalablement à la signature de la convention de recherche avec l'entreprise.

14. PDDS signifie «processus de décision stratégique». 
Cependant, les méthodes de terrain, et, parmi elles, les méthodes qualitatives, sont généralement mieux adaptées pour étudier des processus tel celui de la création d'entreprises (Hernandez, 1999) et pour appréhender la complexité du fonctionnement intime d'une organisation dans son contexte historique et social, une tâche indispensable à la construction de savoirs actionnables pour la gestion des PME.

Pour terminer, examinons les atouts et les difficultés propres aux diverses méthodes qualitatives pour l'élaboration de savoirs actionnables en PME ou en entrepreneuriat.

\subsection{Atouts et difficultés propres aux diverses méthodes qualitatives pour l'élaboration de savoirs actionnables en PME}

Nous n'insisterons pas ici sur l'intérêt que peut présenter pour des chercheurs la recherche en $\mathrm{PME}^{15}$, c'est-à-dire la recherche visant à produire des savoirs actionnables pour la gestion des PME. Nous nous concentrerons plutôt sur la discussion des atouts et des difficultés que présentent les trois méthodes qualitatives types pour la recherche en PME, sachant que, comme l'ont affirmé les dirigeants de PME que nous avons interrogés, en France, la plupart des dirigeants de PME portent peu d'intérêt à la recherche en PME.

\subsubsection{La recherche-intervention: participer in situ à la dialectique faire-connaître}

Par rapport aux autres méthodes qualitatives, la recherche-intervention présente l'intérêt particulier pour les chercheurs de leur permettre de participer à des sortes d'expérimentation in situ et à la mise en acte de la dialectique faire-connaître. Pour des dirigeants de PME, elle présente l'intérêt que des chercheurs en sciences de gestion les aident à concevoir et à mettre en œuvre des réponses taillées sur mesure et argumentées au plan théorique, à des problèmes concrets auxquels ils font face dans la gestion de leur entreprise. À ce titre, on peut dire avec David (2000) que la recherche-intervention est une méthode privilégiée pour développer des savoirs actionnables ${ }^{16}$. Et ce n'est certainement pas un hasard si les exemples que cite Argyris (1993) pour illustrer ses propos sont systématiquement tirés d'opérations de recherche-intervention.

15. Pour une discussion des justifications de cet intérêt, le lecteur peut consulter l'article de Torrès (1997).

16. Pour un exemple de recherche-intervention, voir la contribution de Schmitt dans ce numéro thématique. 


\section{Le «chercheur» : un singulier collectif pour désigner l'équipe de recherche}

Jusqu'à présent, pour éviter d'alourdir le texte, nous avons parlé du chercheur au singulier, comme si un projet de recherche n'impliquait qu'un seul chercheur. En fait, les projets de recherche, surtout lorsqu'ils reposent sur la mise en œuvre de méthodes qualitatives, sont généralement menés par des équipes de chercheurs. Cela permet de répartir la charge de travail, mais aussi d'enrichir les représentations que se construisent les chercheurs et d'accroître leur intersubjectivité à travers le croisement de plusieurs points de vue (Savall et Zardet, 2004), la confrontation d'interprétations diverses et, plus généralement, le travail collectif interactif.

Dans une recherche-intervention, d'autres raisons, liées au fait que les chercheurs sont aussi en position d'intervenant dans l'entreprise, viennent s'ajouter :

- En sa qualité d'intervenant, le chercheur se trouve régulièrement en situation de participer à des discussions de fond ou d'animer des réunions. Dans ces circonstances, il est essentiel qu'un autre chercheur puisse observer et noter les événements qui se produisent au cours des échanges (comportements et réactions de tel ou tel participant, etc.), qui peuvent se révéler capitaux dans la compréhension du phénomène étudié.

- Les problèmes concrets comportent généralement de multiples aspects enchevêtrés: techniques, économiques, politiques, stratégiques, sociaux, culturels, etc. Dans cette perspective, il est intéressant que l'équipe de recherche soit constituée de chercheurs ayant des compétences disciplinaires complémentaires, comme c'est par exemple le cas dans certains laboratoires de recherche qui regroupent des chercheurs de diverses disciplines: sciences de gestion, sciences économiques, sciences du génie (Julien et al., 2003). Cela ne signifie cependant pas qu'une recherche-intervention ne peut se faire qu'au sein d'une vaste équipe multidisciplinaire rompue au travail interdisciplinaire. Certains projets d'ampleur limitée peuvent être menés efficacement dans des équipes de deux ou trois chercheurs.

- À plusieurs chercheurs, il est plus facile de résister à la focalisation habituelle des praticiens sur les problématiques concrètes, pour se ménager du temps pour le travail épistémique et la distanciation du terrain. À cet égard, certains laboratoires de recherche mettent en place des réunions de travail régulières au sein du labo (Savall et Zardet, 1996) ou des «instances de contrôle» (Girin, 1990, p. 170), qui ont pour but, notamment, de renforcer la logique de connaissance en exerçant une pression sur les chercheurs en faveur du travail épistémique et, éventuellement, en les guidant dans ce travail. 
Des dirigeants de PME peuvent s'interroger sur ce qui distingue fondamentalement une recherche-intervention d'une intervention de consultants. Que peut-on leur répondre?

Même si certaines sociétés de conseil effectuent systématiquement des retours d'expérience à l'issue de chaque mission afin de capitaliser les connaissances que les consultants développent au cours de leurs missions, une intervention de consultants n'a pas pour finalité première de construire des savoirs. Sa finalité première est de faire vivre une société commerciale en aidant les entreprises clientes de cette société à améliorer leur performance. La finalité première d'une rechercheintervention n'est pas économique, puisque la plupart des chercheurs en sciences de gestion sont d'abord des enseignants à plein temps. Sa finalité première est d'élaborer des savoirs en participant directement à des actions visant à modifier certaines caractéristiques du fonctionnement des organisations (Savall, 1985, p. 242). De ce fait, dans une recherche-intervention, toute l'intervention est conçue en référence à deux buts indissociables (Verstraete, 1997; Schmitt, Julien et Lachance, 2003) : 1) aider l'entreprise à remédier à une situation problématique ressentie ; 2) élaborer des savoirs actionnables (légitimés), en liaison avec les processus de changement conçus et mis en œuvre en référence au premier but.

Quelles sont les conséquences pour une PME de cette différence de finalités ?

- Une recherche-intervention a généralement une durée beaucoup plus longue (souvent au minimum une année) qu'une intervention de consultants, en raison des travaux théoriques et épistémiques effectués par les chercheurs en relation avec l'intervention et aussi parce que les activités de recherche ne représentent qu'une partie de l'activité professionnelle des enseignants-chercheurs. De ce fait, ils ne peuvent en général participer qu'à une seule recherche-intervention à la fois. Ils ont donc une expérience de l'intervention en entreprises et un professionnalisme nettement moindres que des consultants exerçant ce métier à plein temps depuis de nombreuses années. Cela peut amener le dirigeant d'une PME à penser qu'une recherche-intervention est beaucoup plus hasardeuse pour son entreprise qu'une intervention de consultants et qu'elle prend trop de temps.

- Le coût financier direct d'une recherche-intervention, qui n'est pas négligeable pour une PME, est généralement bien inférieur à celui d'une intervention de consultants. En revanche, dans certaines régions françaises, les PME bénéficient de fonds régionaux d'aide au conseil (FRAC), dont ne peuvent pas profiter les recherches-interventions, ce qui réduit d'autant le différentiel de coût entre les deux types d'intervention. 
Tous ces éléments, combinés au fait qu'en France les dirigeants de PME se sentent encore peu concernés par la recherche en sciences de gestion, rendent difficile pour des chercheurs de trouver des PME qui acceptent de s'engager avec eux dans des recherches-interventions.

\subsubsection{L'étude de cas: un moyen pour intéresser des PME à la recherche en gestion?}

La différence d'apport pour une PME entre une recherche-intervention et une étude de cas peut être exprimée schématiquement comme celle provenant, d'un côté, d'une intervention visant à améliorer une situation problématique ressentie et de l'autre, de la mise en évidence de problèmes latents non nécessairement ressentis. En effet, dans une recherche-intervention, les chercheurs travaillent, entre autres, à l'amélioration d'une situation problématique peut-être mal définie mais tout de même ressentie par l'entreprise au démarrage de la recherche. Une étude de cas, même si ce n'est pas sa finalité première, permet en général au dirigeant de la PME de prendre conscience d'un certain nombre de dysfonctionnements de son entreprise qu'il n'avait pas encore perçus. Cette prise de conscience peut être suscitée par la combinaison de plusieurs éléments : effet de recadrage provenant des représentations renvoyées par les chercheurs en décalage par rapport aux siennes (Plane, 1998); des éléments de comparaison avec d'autres entreprises qui l'interpellent; des questionnements qui éclairent des phénomènes auxquels il n'était pas attentif. Par exemple, dans une étude de cas qui révèle qu'une PME fonctionne sur le mythe: «nous sommes différents, personne ne sait faire ce que nous faisons, nous n'avons donc pas de concurrents », l'insistance des chercheurs à vouloir savoir qui sont les acteurs du secteur, les groupes stratégiques, les compétences fondamentales de l'entreprise, etc., a toutes les chances d'alerter le dirigeant de cette PME sur l'attention insuffisante qu'il porte aux autres acteurs de son secteur et à leurs manœuvres stratégiques.

Une étude de cas est à la fois plus brève et moins coûteuse qu'une rechercheintervention et ne fait pas courir de risques à l'entreprise ; elle est donc plus facile à négocier avec des dirigeants de PME. De ce fait, l'étude de cas peut certainement constituer une voie privilégiée pour amener des dirigeants de PME n'ayant aucune expérience de la recherche en sciences de gestion ou en entrepreneuriat à ouvrir les portes de leur entreprise à des chercheurs.

Une faiblesse de l'étude de cas par rapport à la recherche-intervention est qu'a priori elle ne permet pas au chercheur d'observer in situ la mise en acte de la dialectique faire-connaître, sauf si l'étude de cas se déroule au moment de la mise en œuvre de décisions visant à susciter des changements importants dans la PME considérée. Une manière de contourner cette faiblesse est de s'intéresser à des changements passés, à travers la consultation de documents internes et le recueil 
de narrations. De cette façon, le chercheur peut accéder à une certaine connaissance de la manière dont se sont déroulés divers changements passés et de l'écart qui a existé entre ce qui était attendu et ce qui s'est effectivement produit. Il importe alors qu'il garde présent à l'esprit le fait que les narrations ne sont pas des observations faites en direct, mais des remémorations de personnes ayant été impliquées dans les événements relatés.

Une étude de cas qui permet de relever un certain nombre de problèmes latents peut inciter le dirigeant à poursuivre la collaboration sous la forme d'une recherche-intervention autour du processus d'amélioration de situations problématiques ainsi reconnues. Ainsi, dans l'exemple évoqué précédemment, à l'issue de l'étude de cas, le dirigeant peut décider de poursuivre la collaboration avec les chercheurs en engageant une recherche-intervention autour de la conception / mise en place d'un système de veille stratégique dans cette entreprise.

Par ailleurs, une étude de cas peut conduire à repérer la présence dans l'entreprise de connaissances tacites importantes sur une problématique gestionnaire spécifique. Si celle-ci peut susciter des questions de recherche que les chercheurs jugent intéressantes, ils pourront tenter de négocier avec cette entreprise l'engagement d'un projet de recherche complémentaire qui viserait à transformer, par le biais de récits de pratiques, ces connaissances tacites en savoirs actionnables légitimés.

\subsubsection{Exploiter le récit de pratiques pour co-construire des savoirs actionnables}

La mise en œuvre de la méthode des récits de pratiques peut être envisagée lorsque, dans une entreprise, il existe des connaissances tacites importantes sur une problématique gestionnaire ou managériale particulière (par exemple, l'empowerment des équipes opérationnelles) et que cette entreprise est désireuse de les expliciter. Dans cette méthode, le recueil des informations s'effectue au cours d'entretiens hautement interactifs. L'objectif des entretiens avec les personnes qui sont intervenues sur cette problématique gestionnaire et ont participé à la conception d'artefacts organisationnels destinés à y répondre est de les amener à dépasser la narration de ce qui a été fait afin qu'elles parviennent à expliciter les raisons qui les ont guidées (autrement dit, les pourquoi et les «pour quoi» des comment). Sont également interrogées des personnes qui ont joué un rôle dans la mise en place des artefacts et des personnes qui vivent leur fonctionnement au quotidien. À travers plusieurs itérations d'entretiens avec ces différents acteurs et la confrontation des multiples éclairages recueillis, cette explicitation de connaissances tacites prend la forme d'une co-construction de savoirs actionnables locaux par les praticiens et les chercheurs concernés. Les praticiens interrogés peuvent plus ou moins s'impliquer dans le travail épistémique complémentaire qui vise à transformer ces savoirs locaux en savoirs légitimés. 
Pour une PME, l'explicitation sous forme de savoirs locaux de connaissances tacites existant dans l'entreprise permet en premier lieu à chacun des acteurs concernés, et notamment au dirigeant, « d'évoluer dans la compréhension personnelle de ce qu'il fait» (d'Amboise, 1996, p. 29). Elle facilite également le partage de ces connaissances dans l'entreprise et leur articulation à d'autres connaissances déjà là. Elle permet aussi leur capitalisation et leur mémorisation en vue d'exploitations potentielles ultérieures. Le travail épistémique effectué en vue de la légitimation de ces savoirs permet de les approfondir, voire de les dépasser; il leur apporte une valeur supplémentaire.

La méthode des récits de pratiques fournit au chercheur un accès à des connaissances tacites sur des «faire». Une des limites des autres méthodes de recherche eu égard à l'hypothèse formulée par Vico selon laquelle on ne connaît vraiment que ce que l'on fait (\$2.1) tient au fait que, même dans le cadre d'une recherche-intervention, le chercheur se trouve en position d'extériorité par rapport à l'organisation dans laquelle il mène ses recherches : à la différence des membres de l'organisation impliqués dans les phénomènes organisationnels qu'il étudie, il n'est jamais affecté intimement par ces phénomènes même s'il a contribué à les définir et même s'il accompagne leur mise en œuvre concrète. Recueillir le point de vue de personnes qui vivent intimement ces «faire» peut apporter des éclairages insaisissables autrement par un chercheur - hormis dans le cas exceptionnel et souvent problématique de l'observation participante ${ }^{17}$.

Plus encore qu'une étude de cas, une recherche fondée sur le récit de pratiques peut être brève, peu coûteuse et dénuée de risque pour l'entreprise. Des PME devraient donc pouvoir assez aisément être convaincues de s'engager dans de telles opérations de recherche. Celles-ci ont toutefois un préalable : savoir que dans telle PME il existe des connaissances tacites importantes sur une problématique gestionnaire qui relève du champ de compétences du chercheur et lui paraît intéressante. Cela suppose donc une certaine connaissance préalable de cette PME, que le chercheur peut avoir acquise de diverses manières, notamment comme évoqué dans le §2.3.2, au cours d'une étude de cas antérieure.

Pour terminer, soulignons qu'il est en général plus facile pour le chercheur de conjuguer travail de terrain et travail épistémique dans des recherches menées à partir d'études de cas ou de récits de pratiques, que dans des recherchesinterventions en raison de l'intérêt pressant que, dans ces dernières, les dirigeants de PME portent à la résolution de problèmes concrets, qui tend à détourner l'attention du chercheur du travail épistémique.

17. Pour une discussion des spécificités de l'observation participante et des questions qu'elle soulève, le lecteur peut consulter notamment Wacheux (1996, p. 217-218), Groleau (2003, p. 216-217) ou Savall et Zardet (2004, p. 80-81). 


\section{Conclusion}

Cet article se proposait de discuter les conditions d'élaboration de savoirs actionnables en PME et en entrepreneuriat, pour qu'ils puissent être considérés comme valables dans une conception des sciences de gestion comme des sciences de l'artificiel et donc dans le référentiel épistémologique des constructivismes.

Le terme de légitimation ayant été préféré à celui de validation en raison de la forte connotation de celui-ci, un savoir actionnable légitimé a été défini comme un savoir dont des praticiens sont susceptibles de faire usage pour affronter des problématiques gestionnaires ou managériales qu'ils rencontrent et légitimé par un travail épistémique rigoureux sur le processus d'élaboration de ce savoir et sur le savoir élaboré lui-même. Le processus de légitimation des savoirs est apparu indissociablement lié au processus de construction de ces savoirs lui-même. Un savoir actionnable légitimé a le statut d'hypothèses plausibles.

Aucune méthode de recherche n'est apparue détenir de rôle privilégié dans l'élaboration de savoirs actionnables légitimés, même si la boucle d'apprentissage «faire pour connaître et connaître pour faire », considérée comme essentielle, met en relief l'importance d'un double ancrage des recherches : épistémique et de terrain et donne la primauté aux méthodes qualitatives. En effet, seules ces méthodes permettent de développer une connaissance approfondie des «faire». La recherche monographique, également appelée étude de cas, est apparue comme un moyen privilégié pour impliquer des PME dans des recherches visant à élaborer des savoirs actionnables pour la gestion de PME et/ou en entrepreneuriat. Une recherche monographique peut même ouvrir la voie à des recherches complémentaires s'appuyant sur d'autres méthodes telles que la méthode des récits de pratiques ou la recherche-intervention.

La principale conclusion de cette réflexion est certainement que toute recherche visant l'élaboration de savoirs légitimés pour la gestion d'entreprises exige un travail épistémique continuel et rigoureux. Ainsi, élaborer des savoirs actionnables légitimés en sciences de gestion conçues comme des sciences d'ingénierie des organisations socioéconomiques, c'est essentiellement transformer de l'expérience en science avec conscience.

\section{Bibliographie}

Allard-Poesi, F. et V. Perret (2003), «La recherche-action», dans Y. Giordano (dir.), Conduire un projet de recherche. Une perspective qualitative, Paris, Éditions EMS, p. 85-132.

Allison, G.T. (1971), Essence of Decision : Explaining the Cuban Missiles Crisis, Boston, Little, Brown \& Co. 
ARGYRIS, C. (1993), Knowledge for Action : A Guide to Overcoming Barriers to Organizational Change, San Francisco, Jossey-Bass ; traduction française : Savoir pour agir, Paris, InterÉditions, 1995.

Argyris, C., R. Putnam et D. MCLAin Smith (1985), Action Science : Concepts, Methods and Skills for Research and Intervention, San Francisco, Jossey-Bass.

AVENIER, M.-J. (1985), «Complémentarité et fragilité des méthodes de recherche en gestion », dans Méthodologies fondamentales en gestion, actes du colloque CNRSFNEGE-ISEOR, novembre, p. 23-52.

AVENIER, M.-J. (1989), « “Méthodes de terrain” et recherche en management stratégique », Sciences de gestion, $\mathrm{n}^{\circ} 14$, p. 199-218.

AVEnIER, M.-J. (1997), La stratégie chemin faisant, Paris, Economica.

Avenier, M.-J. (2002), «Comment des praticiens voient-ils la recherche en sciences de gestion?», Document de recherche Euristik no 4/2002, Lyon, Université de Lyon III, 69 p.

Avenier, M.-J. et C. Pellegrin (2004), Beauvais International : l'intimité client, Paris, Centrale des cas et médias pédagogiques; site Internet: <www.ccip.fr/ccmp/ detail.asp ?id=G1273>.

BLANCO, S. et H. LESCA (2002), «Contribution à la capacité d'anticipation des entreprises par la sensibilisation aux signaux faibles », VI ${ }^{\mathrm{e}}$ CIFPME 2002, Montréal, octobre.

CALORI, R. (2001), «De la connaissance collective ordinaire à la théorie », dans A.C. Martinet et R.A. Thiétart (dir.), Stratégies. Actualité et futurs de la recherche, Paris, Vuibert, p. 85-98.

Chanal, V., H. Lesca et A.C. Martinet (1997), «Vers une ingénierie de le recherche en sciences de gestion », Revue française de gestion, n ${ }^{\circ}$ 116, p. 41-51.

Crozier, M. et E. Friedberg (1977), L'acteur et le système, Paris, Seuil.

DAFT, R.L. et A.Y. LEWIN (1990), «Can organization studies begin to break out of the normal science straight jacket? An editorial essay », Organization Science, vol. 1, $\mathrm{n}^{\mathrm{o}} 1$, p. 1-9.

D’Amboise, G. (1996), «La recherche en gestion des PME: des paradigmes en évolution », Cahier de recherche 96-11-08, Montréal, Chaire d'entrepreneurship McLean Hunter, HEC, 33 p.

DAVID, A. (2000), «La recherche-intervention, cadre général pour la recherche en management », dans A. David, A. Hatchuel et R. Laufer, Les nouvelles fondations des sciences de gestion, Paris, Vuibert, p. 193-213.

DAVID, A., A. HATCHUEL et R. LAUFER (2000), Les nouvelles fondations des sciences de gestion, Paris, Vuibert.

DÉRY, R. (1997), «Topographie épistémologique du champ de recherche en stratégie d'entreprise », Management international, vol. 2, nº 1, p. 11-18.

Dewey, J. (1993), Logique. La théorie de l'enquête, Paris, Presses universitaires de France ; traduction de Logic. The Theory of Inquiry, New York, Henry Holt \& Cy, 1938.

Revue internationale P.M.E., vol. 17, nºs 3-4, 2004

(C) 2004 - Presses de l'Université du Québec

Édifice Le Delta I, 2875, boul. Laurier, bureau 450, Sainte-Foy, Québec G1V 2M2 • Tél. : (418) 657-4399 - www.puq.ca 
FILION, L.J. (1991), Vision et relations : Clefs du succès de l'entrepreneur, Montréal, Les éditions de l'Entrepreneur.

GiordANO, Y. (2003), «Les spécificités des recherches qualitatives», dans Y. Giordano (dir.), Conduire un projet de recherche. Une perspective qualitative, Paris, Éditions EMS, p. 11-39.

GIRIN, J. (1981), «Quel paradigme pour la recherche en gestion?», Sciences de gestion, $\mathrm{n}^{\circ} 2$.

GIRIN, J. (1990), « Analyse empirique des situations de gestion : éléments de théorie et de méthode», dans A.C. Martinet (dir.), Épistémologie et sciences de gestion, Paris, Economica, p. 141-182.

GIROD-SÉviLLE, M. et V. PERRET (1999), «Fondements épistémologiques de la recherche», dans R.A. Thiétart et collab., Méthodes de recherche en management, Paris, Dunod, 1999, p. 13-33.

Giroux, N. (2003), «L'étude de cas», dans Y. Giordano (dir.), Conduire un projet de recherche. Une perspective qualitative, Paris, Éditions EMS, p. 41-84.

Groleau, C. (2003), «L'observation», dans Y. Giordano (dir.), Conduire un projet de recherche. Une perspective qualitative, Paris, Éditions EMS, p. 211-244.

HAFSI, T. (1997), «Le champ de la recherche en stratégie: à la recherche d'un bâton d'aveugle », Management international, vol. 2, n 1, p. 19-26.

Hamel, J., S. Dufour et D. Fortin (1993), Case Study Methods, Newbury Park, Cal., Sage.

HERNANDEZ, E.-M. (1999), « Modèles d'entrepreneuriat : vers une approche contingente et processuelle », Sciences de gestion, $\mathrm{n}^{\circ}$ 26-27, p. 505-526.

Hlady Rispal, M. (2002), La méthode des cas. Applications à la recherche en gestion, Bruxelles, De Boeck Université.

JELINEK, M. et J.A. LitTERER (1995), «Toward entrepreneurial organization : meeting ambiguity with engagement », Entrepreneurship Theory and Practice, vol. 19, $\mathrm{n}^{\circ} 3$, p. 137-167.

Julien, P.-A. et M. MARChESNAY (1992), «Des procédures aux processus stratégiques dans les PME », Piccola Impresa, $\mathrm{n}^{\circ} 1$.

Julien, P.-A., L. RAymond, R. JACOB et G. Abdul-Nour (2003), L'entreprise-réseau, Sainte Foy, Presses de l'Université du Québec.

LEE, A.S. (1989a), «Case studies as natural experiments », Human Relations, vol. 42, nº 2, p. $117-137$.

LEE, A.S. (1989b), «A scientific methodology for M.I.S. Case studies », M.I.S. Quarterly, mars, p. 33-50.

LEE, A.S. (1991), «Integrating positivist and interpretive approaches to organizational research », Organizational Science, ${ }^{\circ}$ 2, vol. 24, p. 342-365.

Legroux, J. (1981), De l'information à la connaissance, Maurecourt, Éditions Mésonnance. 
LE Moigne, J.L. (1990), «Épistémologies constructivistes et sciences de l'organisation », dans A.C. Martinet (dir.), Épistémologie et sciences de gestion, Paris, Economica, p. 81-140.

Le Moigne, J.L. (1995), Les épistémologies constructivistes, Paris, Presses universitaires de France, coll. «Que sais-je ?», no 2969.

LE Moigne, J.L. (2000), «Préface», dans M.-J. Avenier (dir.), Ingénierie des pratiques collectives. La cordée et le quatuor, Paris, L'Harmattan, p. 7-15.

Le Moigne, J.L. (2001 et 2002), Le constructivisme, tomes 1 et 2, Paris, L'Harmattan.

LEWIN, K. (1946), «Action research and minority problems », Journal of Social Issues, $\mathrm{n}^{\circ} 2$, p. 34-46.

MAFFeSOLI, M. (1985), La connaissance ordinaire, Paris, Méridien.

MARCheSnAY, M. (2002), «Les PME de terroir : entre Géo et Clio stratégies », XI ${ }^{\mathrm{e}}$ Conférence de 1'AIMS, ESCP-EAP, Paris ; site Internet : 〈www.stratégie-aims.com>.

MARTINET, A.C. (dir.) (1990), Épistémologie et sciences de gestion, Paris, Economica.

MARTinet, A.C. (1997), «Pensée stratégique et rationalités : un examen épistémologique », Management international, vol. 2, no 1, automne, p. 67-75.

MARTINET, A.C. (2000), «Épistémologie de la connaissance praticable : exigences et vertus de l'indiscipline », dans A. David et al. (dir.), Les nouvelles fondations des sciences de gestion, Paris, Vuibert.

MARTinet, A.C. (2002), «Identification du contexte d'une organisation dans une perspective de management : propos d'étape », Communication présentée au Congrès de l'Union européenne de systémique, Héraklion, octobre.

M'BENGUE, A. (2001), «Posture paradigmatique et recherche en management stratégique », dans A.C. Martinet et R.A. Thiétart (dir.), Stratégies. Actualité et futurs de la recherche, Paris, Vuibert, p. 43-53.

MintzBerG, H. et J. WATERS (1985), « Of strategies, deliberate and emergent », Strategic Management Journal, vol. 6, p. 257-272.

MoISDON, J.C. (1984), « Recherche en gestion et intervention », Revue française de gestion, septembre-octobre, p. 61-73.

NONAKA, I. (1994), « A dynamic theory of organizational knowledge creation », Organization Science, vol. 5, no 1, p. 14-37.

PATUREL, R. et H. SAVALl (2001), « La légitimité de la recherche académique en management stratégique », Revue Sciences de gestion, $\mathrm{n}^{\circ}$ 29, p. 175-208

Piaget, J. (1967), Logique et connaissance scientifique, Paris, Gallimard, Encyclopédie de la Pléiade.

Plane, J.M. (1998), «Pour une approche ethnométhodologique de la PME», Revue internationale PME, vol. 11, $\mathrm{n}^{\circ} 1$.

Plane, J.M. (2000), Méthodes de recherche-intervention en management, Paris, L'Harmattan.

Rouleau, L. (2003), «La méthode biographique », dans Y. Giordano (dir.), Conduire un projet de recherche. Une perspective qualitative, Paris, Éditions EMS.

Revue internationale P.M.E., vol. 17, nos 3-4, 2004

(C) 2004 - Presses de l'Université du Québec

Édifice Le Delta I, 2875, boul. Laurier, bureau 450, Sainte-Foy, Québec G1V 2M2 • Tél. : (418) 657-4399 - www.puq.ca 
SaVall, H. (1985), «Où va la recherche en sciences de gestion? », Revue française de gestion, $\mathrm{n}^{\circ}$ 53-54, p. 242-253.

SAVAlL, H. et V. ZARDET (1996), «La dimension cognitive de la recherche-intervention: la production de connaissances par interactivité cognitive», Revue internationale de systémique, vol. 10, $\mathrm{n}^{\text {os }}$ 1-2, p. 157-189.

SAVALl, H. et V.ZARDET (2004), Recherche en sciences de gestion : approche qualimétrique, Paris, Economica.

Schmitt, C., P.-A. Julien et R. Lachance (2003), «Pour une lecture des problèmes complexes en PME : approche conceptuelle et expérimentation », dans P.-A. Julien, L. Raymond, R. Jacob et G. Abdul-Nour, L'Entreprise-Réseau, Sainte-Foy, Presses de l'Université du Québec, p. 221-250.

SimON, H.A. (1969/2004), The Sciences of the Artificial, The MIT Press; traduction française de la 3e édition: Sciences de l'artificiel, Paris, Gallimard, 2004.

Simon, H.A. (1983), Reason in Human Affairs, Stanford, Stanford University Press.

THIÉTART, R.A. (2001), «Management et complexité : concepts et théories », dans A.C. Martinet et R.A. Thiétart (dir.), Stratégies. Actualité et futurs de la recherche, Paris, Vuibert, p. 361-375.

THIÉTART, R.A. et collab. (1999), Méthodes de recherche en management, Paris, Dunod.

TORRÈs, O. (1997), «Pour une approche contingente de la spécificité de la PME », Revue internationale PME, vol. $10, \mathrm{n}^{\circ} 2$.

Verstraete, T. (1997), «Cartographie cognitive et accompagnement du créateur d'entreprise », Revue internationale PME, vol. 10, n 1 .

VIco, G. (1981), La vie de G. Vico écrite par Vico lui-même, Paris, Grasset; traduit de La méthode des études de notre temps, 1708.

WACHEUX, F. (1996), Méthodes qualitatives et recherche en gestion, Paris, Economica.

YIN, R.K. (1984), Case Study Research, Design and Methods, Newbury Park, Cal., Sage Publications. 